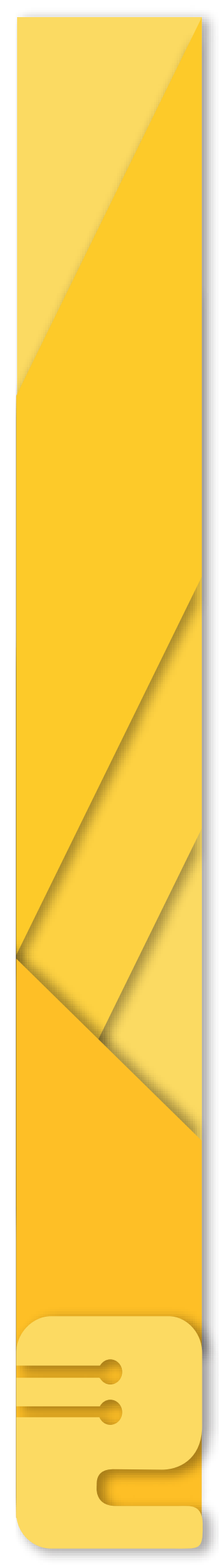

\title{
APROVECHAMIENTO DE RESIDUOS VEGETALES DE PÉTALOS DE ROSAS, TALLOS DE GIRASOL Y VÁSTAGO DE PLÁTANO PARA LA FABRICACIÓN ARTESANAL DE PAPEL ${ }^{1}$
}

\author{
HARVESTING OF PETAL WASTE FROM ROSES, SUNFLOWER STALKS AND BANANA \\ SHOOTS FOR MANUFACTUE HANDMANDE OF PAPER \\ APROVEITAMENTO DE RESÍDUOS VEGETAIS DE PÉTALAS DE ROSAS, CAULE DE GIRASSOL \\ E RAMO DO PLÁTANO PARA A FABRICAÇÃO ARTESANAL DE PAPEL
}

\author{
Freddy Alfonso Moreno², Marlen Robayo Quintana³, Laura Ferrucho Rodríguez ${ }^{3}$, \\ Mónica Vargas Oyola ${ }^{3}$
}

Fecha de recibido: Febrero 1 de 2016 | Fecha de aprobado: Marzo 28 de 2016

\section{Resumen}

Este artículo pretende brindar una alternativa para la fabricación de papel artesanal, con características similares al que se obtiene a partir de la celulosa extraída de la corteza de los árboles, a través del aprovechamiento de los residuos vegetales que contienen altos porcentajes de celulosa tales como pétalos de rosas, vástago de plátano y tallos de girasol, que se producen en las plazas de mercado en la ciudad de Bogotá. Se ha considerado que, de los 70,5 T/día de residuos sólidos que genera la ciudad, $88,5 \%$ son residuos vegetales de frutas, verduras y hortalizas que no son aprovechados.

El procedimiento establecido permitió fabricar hojas de papel mezclando diferentes porcentajes de cada residuo vegetal, triturado y molido con pulpa de papel reciclado, para establecer cuáles de ellos ofrecían mejores características de calidad respecto al color, estabilidad dimensional, pliegue, escritura, humectación al ambiente, textura y resistencia a la penetración. Los impactos de este proyecto están dirigidos a contribuir en la búsqueda e implementación de técnicas

1 Proyecto adelantado como parte de la investigación titulada Obtención de pulpa de papel a partir de residuos orgánicos, financiado mediante la Tercera Convocatoria para el Desarrollo y Fortalecimiento de la Investigación en UNIMINUTO, realizada por la Dirección General de Investigaciones y desarrollado por los semilleros de investigación Gestión Integral de Residuos Sólidos del Programa de Ingeniería Agroecológica y el semillero en Producción y Uso de las Energías Alternativas y Renovables en Procesos Industriales (Dipiera) del Programa de Ingeniería Industrial.

2 Qco. Mg. Ing. Docente del Programa de Ingeniería Industrial de la Corporación Universitaria Minuto de Dios - UNIMINUTO, Bogotá-Colombia. Miembro del semillero de investigación: producción y uso de las energías alternativas y renovables en la industria - DIPIERA.

flamoreno17@yahoo.com.ar, falfonso@uniminuto.edu.

3 Estudiantes de Ingeniería Industrial -Programa de Ingeniería Industrial, Corporación Universitaria Minuto de Dios - UNIMINUTO, Bogotá - Colombia, flamoreno17@yahoo.com.ar 
sustentables de producción con el aprovechamiento de los residuos orgánicos generados en grandes cantidades en los centros de acopio de alimentos, la generación de ideas de negocios inclusivos -ya que se pretende favorecer a personas en condiciones de vulnerabilidad como desplazamiento y mujeres cabeza de familia-, además de incentivar la cultura del reciclaje y fortalecer la cadena productiva de este sector, permitiendo su restablecimiento socioeconómico a través del desarrollo de una actividad que exige una capacitación mínima para su desempeño.

Palabras clave: papel reciclado, licuar, prensado, secado, triturar, vástago.

\section{Abstract}

This article aims to provide an alternative for making handmade paper with characteristics similar to that obtained from the cellulose extracted from the bark of the trees, through the use of plant residues containing high percentages of cellulose as rose petals scion of banana and sunflower stalks, occurring in the market places in the city of Bogotá, considering that $70.5 \mathrm{~T} /$ day of solid waste generated by the city $88.5 \%$ are fruit vegetable waste, vegetables that are not exploited.

The procedure established allowed to manufacture sheets of paper by mixing different percentages of each plant debris crushed and ground with recycled paper pulp to establish which of them offered better quality characteristics regarding color, dimensional stability, crease, writing, wetting the environment, texture and penetration resistance. The impacts of this project are aimed at contributing to the search and implementation of sustainable production techniques with the use of organic waste generated in large quantities in the collection centers for food, idea generation inclusive business as it seeks to promote people in vulnerable as displacement and female heads of household, in addition to encouraging the recycling culture and strengthen the productive chain of the sector; allowing its socio-economic recovery through the development of an activity that requires minimal training to be performance.

Keywords: recycled paper, liquify, pressing, drying, grinding, vástago.

\section{Resumo:}

Este artigo pretende brindar uma alternativa para a fabricação de papel artesanal, com características similares ao que se obtém a partir da celulose extraída da corteza das árvores, através do aproveitamento dos resíduos vegetais que contêm altas percentagens de celulose tais como pétalas de rosas, ramo de plátano e caule de girassol, que se produzem nas praças de mercado na cidade de Bogotá. Considerou-se que, dos 70,5 T/dia de resíduos sólidos que gera a cidade, 88,5\% são resíduos vegetais das frutas, verduras e hortaliças que não são aproveitados.

O procedimento estabelecido permitiu fabricar folhas de papel misturando diferentes percentagens da cada resíduo vegetal, triturado e molido com polpa de papel reciclado, para estabelecer quais deles ofereciam melhores características de qualidade com respeito à cor, estabilidade dimensional, dobra, escritura, humedecimento ao ambiente, textura e resistência à penetração. Os impactos deste projeto estão dirigidos a contribuir na busca e implementação de técnicas sustentáveis de produção com o aproveitamento dos resíduos orgânicos gerados em grandes quantidades nos centros de recolha de alimentos, a geração de ideias de negócios inclusivos -já que pretende-se favorecer a pessoas em condições de vulnerabilidade como deslocação e mulheres cabeça de família-, além de incentivar a cultura do reciclagem e fortalecer a corrente produtiva deste setor, permitindo sua restauração socioeconômica através do desenvolvimento de uma atividade que exige uma capacitação mínima para seu desempenho.

Palavras-chave: papel reciclado, liquefazer, prensagem, secagem, triturar, ramo. 


\section{INTRODUCCIÓN}

Bogotá tiene 36 plazas de mercado -18 de propiedad del Distrito y 18 de carácter privado- donde se producen diariamente $70.5 \mathrm{~T} /$ día, contribuyendo con el $1.4 \%$ de los residuos totales de la ciudad; el $88.5 \%$ corresponde a residuos vegetales -verduras, frutas y hortalizas- equivalente a 62.4 toneladas, 11.5 se producen en las plazas de mercado y 50.9 en Corabastos. La descarga de frutas, verduras, hierbas, plátano y hortalizas es la actividad que genera mayor cantidad de residuos. Los transportadores evidentemente no se llevan los desechos y estos quedan abandonados en el sitio de descarga. En Corabastos el nivel de pérdida de productos vegetales es del $2 \%$-aproximadamente $18500 \mathrm{~T} /$ año-. El nivel de pérdidas en las plazas de mercado oscila entre el 5 y 7\%. (Uaesp, 2013).

La mayoría de estos residuos han sido caracterizados por sus altos niveles de celulosa y tienen potencial para ser empleados como sustitutos de la fibra virgen de la madera, la cual es la principal fuente de materia prima de la industria del papel. Actualmente sólo un $9 \%$ de los materiales utilizados para la fabricación de papel a nivel mundial procede de fuentes no madereras: paja de arroz, trigo, bagazo de caña de azúcar, cáñamo, algodón, kenaf, etc. (Canché-Escamilla, De los SantosHernández, Andrade-Canto, Gómez-Cruz, 2015). El resto del papel se produce a partir de fibra virgen en un $55 \%$ y fibra en un reciclada $38 \%$, pero las fuentes de fibra procedentes del papel recuperado y las fibras de origen agrícola aún no se están aprovechando en su máximo potencial. (Ervasti, Miranda, Karaunen, 2016; Ministerio Agricultura, 2008).

Para producir una tonelada de papel para impresión la industria requiere las siguientes cantidades de materias primas y energía:

- $1845,10 \mathrm{~kg}$ de madera.

- $108,06 \mathrm{~kg} \mathrm{CaO}$.

- $180,11 \mathrm{~kg}$ de sulfato de sodio.

- 38,02 kg de carbonato de sodio anhidro.

- Aditivos como el almidón.

- Resinas, alumbre, dióxido de titanio.

- Bentonita, cascina.

- 100114761 I de agua.

- 30,86 millones de вUт de energía.

Deshechos:

- 42,02 kg contaminantes del aire

- $18,01 \mathrm{~kg}$ contaminantes del agua

- $88,05 \mathrm{~kg}$ de residuos sólidos. (Ervasti, et al., 2016).
La tala de árboles contribuye al cambio del equilibrio climático, lo que ha traído consecuencias como el calentamiento global; por lo menos el $10 \%$ de este fenómeno ha sido causado por el ejercicio de tala. Considerando que los árboles son los organismos vivos que fabrican el agua y la recogen mediante un proceso natural para reciclar la humedad a través de sus hojas, además de absorber el calor solar y purificar el aire mientras absorben el dióxido de carbono, al reducirse la vegetación en una cuenca no hay nada que retenga la lluvia arrastrando las capas de suelo y provocando, de ese modo, la erosión del suelo. (Burgos, 2012; Otero, 2001).

Colombia no es la excepción en estas estadísticas: según cifras del DANE (2012) y la Cámara de la Industria de Pulpa, Papel y Cartón de la ANDI, se consumieron durante el 2012 alrededor de 760607 toneladas de papel y cartón. Para el primer semestre del mismo periodo se reportó un consumo de papeles de impresión de 220 mil toneladas -materia de la industria gráfica colombiana-, lo que marcó un crecimiento de 2,8\%. (Observatorio Colombiano de Ciencia y Tecnología. 2013).

Es importante buscar nuevas alternativas de obtención de materias primas para la fabricación de papel con características similares a las del papel que se obtiene a partir de la celulosa. Una de estas alternativas es la utilización de residuos sólidos orgánicos, entre ellos el mismo papel que ya ha sido utilizado, y el componente de fibra que posee la celulosa de los árboles lo aportan residuos provenientes de desechos agrícolas, por ejemplo: cáscaras, bagazo, tusa, u otras plantas.

Para el año 2012 tan solo en los hogares de Bogotá se produjeron 1040496 toneladas métricas de residuos sólidos. Diariamente en la ciudad se producen 6200 toneladas y en fechas de fiestas esta cantidad se eleva entre 8000 y 9000 toneladas. Se estima que alrededor del $70 \%$ de estos residuos son de origen orgánico y todo va al relleno sanitario de Doña Juana. (Uaesp, 2013).

Este relleno sanitario está técnicamente construido para depositar las basuras. Los residuos sólidos orgánicos en su proceso de descomposición generan gases, malos olores y lixiviados; sin embargo, la capacidad para tratar estos líquidos es de $16 \mathrm{~L} / \mathrm{s}$, valor insuficiente para el volumen de residuos arrojados en este lugar. Por esto, al no poder ser tratados en su totalidad, tales residuos se vierten 
en ríos aledaños como el Tunjuelito (Uaesp, 2013). Esta situación hace necesario pensar en plantear soluciones e implementar estrategias y mecanismos que permitan el máximo aprovechamiento de estos residuos para ayudar a mitigar este impacto. El potencial de aprovechamiento de los residuos en Colombia es muy alto porque reincorpora al ciclo productivo aproximadamente un $10 \%$ de los residuos sólidos generados. (Rodríguez, Londoño y Herrera, 2008).

Por esto el proyecto plantea la fabricación de papel como alternativa para aprovechar los residuos vegetales de pétalos de rosas, vástago de plátano y tallos de girasol, producidos en las plazas de mercado, mezclándolos con proporciones en peso de $20 \%$ al $40 \%$ en pulpa de papel reciclado acorde con los procedimientos planteados a nivel mundial en pequeñas y medianas industrias de Estados Unidos, Europa y Japón; se obtiene de este modo un producto de calidad y potencial uso en productos artesanales de manualidad y escritura.

\section{MARCO TÉCNICO}

\section{Residuos sólidos orgánicos}

Son restos de productos biodegradables tales como porciones de comida, frutas, flores, verduras, carne y huevos. Estos materiales son los desechos típicos y comunes de los centros de acopio de alimentos como las plazas de mercado presentes en los puntos de concentración poblacional. Los materiales plásticos no ingresan en este grupo a pesar de estar constituidos por moléculas orgánicas muy complejas que requieren procesos muy específicos para su degradación. (Burgos, 2012).

Los residuos orgánicos se encuentran en diversos ambientes y su producción es evaluada en miles de toneladas anuales en las grandes ciudades. El manejo y disposición de ellos es un problema ambiental, social, económico y político debido a que todas las instituciones públicas y privadas como papelerías, hospitales, restaurantes, bodegas, hogares y centros de abastecimiento de alimentos los generan en diversas cantidades. (Pintado, Joaquín \& Grande, 2010).

\section{Pulpa de papel usado - papel reciclado-}

Con el propósito de ahorrar materias primas y energía, el papel puede ser reciclado reutilizando las fibras del papel usado en lugar de las fibras de madera. Este papel usado debe ser preparado previamente antes de aplicarse a la máquina de papel.

Se produce una suspensión bombeable a partir de la entrada de material en un recipiente lleno de agua y papel usado. En este recipiente se rompe el papel usado en fibras por disolución en agua. Las partículas de tinta y las impurezas - tales como papel de aluminio, productos textiles, bolsas de plástico, piedras, grapas o trozos de madera- se separan de la suspensión antes de que se pueda aplicar a la máquina de papel. (Pérez, Rojas y Ordóñez, 2010).

Las impurezas que se eliminan durante el proceso de reciclado - rechazo- se pueden llevar a calderas para combustión y así generar energía térmica. (Burgos, 2012).

\section{Reuso frente a la incineración}

El reciclado de papel contribuye a disminuir el uso de fibra generada directamente de árboles talados, sin embargo siempre es necesario el aporte de nuevas fibras de los bosques en el ciclo del papel. Los residuos de papel contienen una gran cantidad de fibras rotas que no pueden volver a ser procesadas. En cada ciclo de reprocesamiento, el $10-20 \%$ de las fibras son demasiado pequeñas para su reutilización y tienen que ser sustituidas. (Burgos, 2012).

El papel que no se puede reciclar más se puede combustionar junto con otros residuos domésticos en los incineradores encontrados a nivel industrial. El papel influye positivamente en el proceso de incineración, ya que se quema fácilmente $y$, por tanto, reduce la demanda suplementaria de los combustibles fósiles. A modo de ejemplo, la combustión de una tonelada de papel usado sustituye aproximadamente 600 litros de petróleo. (Wang, Templer \& Murphy, 2012).

Dado que los incineradores municipales comúnmente generan energía, por ejemplo, vapor para sistemas 
de calefacción y electricidad, la incineración de residuos de papel que no pueden ser reciclados o reusados en otras materias más veces es una manera de recuperar energía.

\section{Papel reciclado y el uso de fibras naturales}

La producción de papel a nivel industrial genera numerosos efectos medioambientales, y por esto expertos europeos y estadounidenses indican que el papel fabricado a partir de fibras recicladas es menos nocivo para la naturaleza que el papel producido a partir de fibras vírgenes (Burgos, 2012). Los impactos sobre el medioambiente son los siguientes:

- Emisión de gases de efecto invernadero -GEI-: dióxido de carbono $\left(\mathrm{CO}_{2}\right)$ y metano $\left(\mathrm{CH}_{4}\right)$ que contribuyen al cambio climático pues permiten que el calor se concentre en la atmósfera terrestre

- Emisión de partículas: las partículas pequeñas (< $10 \mu \mathrm{m})$ que se dispersan en la atmósfera durante la combustión pueden causar asma y otras enfermedades respiratorias e incluso cáncer por inhalación de las mismas.

- Emisión de dióxido de azufre $\left(\mathrm{SO}_{2}\right)$ : resulta de la combustión de combustibles que contienen azufre - carbón, petróleo- en las calderas y produce problemas de contaminación atmosférica como la lluvia ácida o el esmog.

- Demanda Química de Oxígeno -DQO-: indica la cantidad de materia orgánica que persiste en los efluentes de agua.

- Demanda Bioquímica de Oxígeno -DBO-: establece la cantidad de oxígeno consumida por los microorganismos al degradar la materia orgánica del efluente. Verter efluentes con un alto valor de DBO puede provocar una reducción del oxígeno disuelto en el agua y afectar negativamente a los peces y otros organismos.

- Halógenos orgánicos absorbibles -HOA-: son una medida indirecta de compuestos orgánicos clorados, algunos de los cuales son tóxicos.

En el ciclo de fabricación del papel la eficiencia energética es un factor importante pues la recuperación de fibras propone un consumo menor de energía; sin embargo, el uso de combustibles de origen fósil es común en este proceso industrial. Una tonelada de papel producida a partir de fibras recicladas consume aproximadamente $2 \mathrm{mwH}$, es decir, un $40 \%$ menos energía que el papel producido a partir de fibras nuevas. Esta cantidad de energía se corresponde con la energía que consume un hogar europeo en un mes y medio. (Wang et al., 2012)
El potencial promedio de emisiones de dióxido de carbono evitadas es de $700 \mathrm{~kg}$ por tonelada de papel reciclado, en concordancia con el papel producido a partir de fibras nuevas. (Canché-Escamilla et al., 2015).

\section{MATERIALES Y MÉTODOS}

Los residuos orgánicos - vástago de plátano, pétalos de rosa y tallos de girasol- procesados para la fabricación de la pulpa para papel fueron recolectados de las plazas de mercado y floristerías presentes en la localidad de Engativá -Bogotá, Colombia-; estos residuos fueron almacenados en una bodega aireada para evitar la degradación por exceso de humedad espacio prestado por la unidad ambiental de la sede principal de UNIMINUTO- para ser manipulados de manera conveniente como se indica.

Trituración del residuo orgánico: para esto es necesario emplear una trituradora de tipo semiindustrial -verificar que la salida de esta no se encuentre obstruida o tenga residuos de algún elemento, este procedimiento se debe realizar estando apagada-. Se trituran 1000 gramos de vástago de plátano o tallos de girasol. Figura 1.

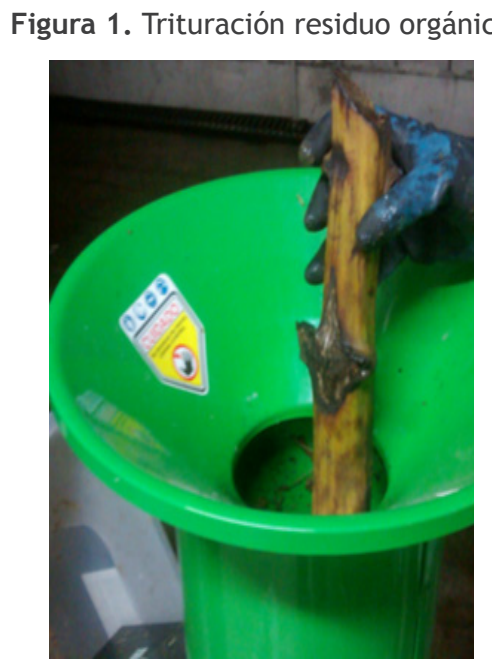

Fuente: creación autores (2014).

Para garantizar una trituración más fina es necesario que se licúe en una licuadora de uso doméstico. Este proceso se realiza con los bagazos de plátano, tallo de girasol y pétalos de rosas tratando cada uno por separado.

Cocción del material triturado y licuado de los residuos orgánicos -bagazo de plátano y tallo de girasol-: para ello se utilizan 6 litros de agua y se 
aplican $30 \mathrm{~g}$ de hidróxido de sodio $(\mathrm{NaOH})$. Este proceso dura aproximadamente una hora. Figura 2. (Canché-Escamilla et al., 2015).

Los residuos degradados alcalinamente son lavados para ser neutralizados con adiciones de ácido cítrico hasta pH 7.0 para minimizar la contaminación por el uso de sustancias no biodegradables; las fibras son recogidas y procesadas en una licuadora durante 5 minutos.

Figura 2. Cocción residuo triturado

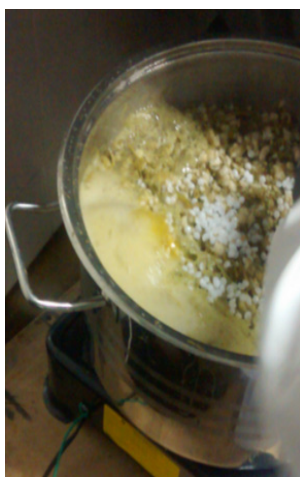

Fuente: creación autores (2014).

Mezclado de la pulpa: en diez litros de agua se combina la pulpa del residuo orgánico, procesado con proporciones de $0 \%, 20 \%$ y $40 \%$ en peso de pulpa de papel blanco reciclado, obtenido de manera semejante respecto a la trituración y molido en licuadora sin cocción alcalina. Figura 3.

Figura 3. Mezclado de la pulpa

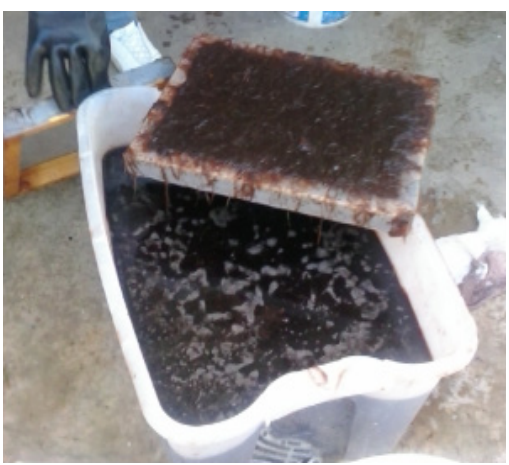

Fuente: creación autores (2014).

Una vez se obtiene esta mezcla se tamiza con una malla de alambre para formar la hoja de papel que se ubica en una tela de banner y se retira el exceso de agua con una esponja. Figura 4.
Figura 4. Eliminación del exceso de agua

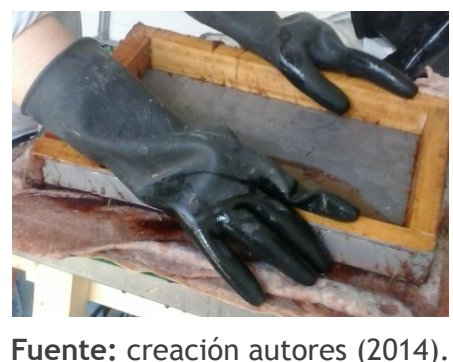

Prensado: después de retirado el tamiz se ubica un fieltro encima de la hoja formada el cual servirá de cubierta para impedir que las fibras se separen en el momento de hacer la compresión. Figura 5.

Figura 5. Prensado de las hojas de papel formadas

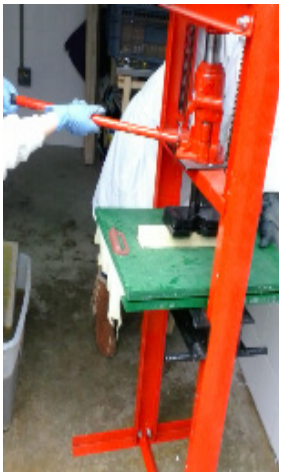

Fuente: creación autores (2014).

Secado: eliminado el exceso de agua en la prensa se retira el fieltro que cubre la hoja formada. Figura 6.

Figura 6. Retirado del fieltro

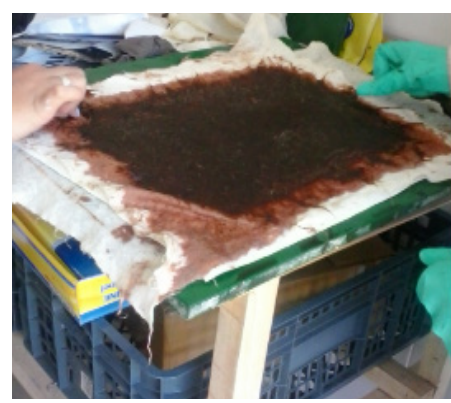

Fuente: creación autores (2014).

El secado tiene una duración de tres días y se puede hacer al aire sin influencia directa de la radiación solar ubicando las hojas de forma vertical en soportes cubiertos por las mallas de alambre. Figura 7. 
Figura 7. Secado vertical por aire

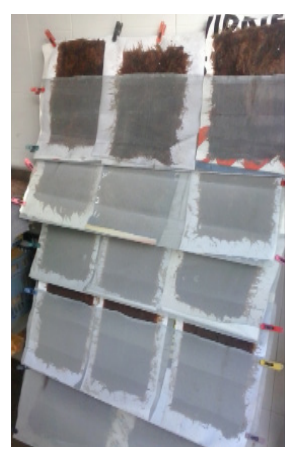

Fuente: creación autores (2014).

Medición de las propiedades de calidad del papel obtenido: se analizaron las hojas hechas con los residuos de pétalos de rosas, tallos de girasol y vástago de plátano evaluando los siguientes aspectos: color, estabilidad dimensional, pliegue, escritura, humectación al ambiente, textura y resistencia a la penetración. Para ello se asignó una calificación mínima y máxima de 1 a 5 según se ajustaba, por comparación observada, al papel bond tradicional encontrado en el mercado (González, 2005). Cada parámetro se midió de la siguiente forma:

Color: 1 es blanco y 5 oscuro con respecto al color del residuo orgánico original.

Estabilidad dimensional: facilidad para dejarse doblar, 1 es fácil de doblar y 5 no se dobla.

Pliegue: hasta donde permite doblar, 1 marca mucho los dobleces y 5 se rompe al doblar.

Escritura: permite dejarse escribir, 1 deja escribir igual a hoja en blanco y 5 no permite escribir con suavidad.
Humectación al ambiente: ganancia de peso del papel por adquirir humedad del ambiente.

1 entre $0-20 \%$; 2 entre $20-40 ; 3$ entre $40-60 \%$; 4 entre $60-80 \%$ y 5 entre $80-100$.

Textura: qué tan áspera es la hoja, 1 es lisa como el papel blanco y 5 es áspera grado lija gruesa.

Resistencia a la penetración: 1 no penetra, soporta peso, y 5 penetra fácil, no soporta el peso. Se ha tomado como referencia una hoja blanca tamaño carta de gramos tamaño $28 \times 12,5 \mathrm{~cm}$.

\section{RESULTADOS Y DISCUSIÓN}

El procedimiento establecido para la fabricación de papel fue determinado tratando los residuos de pétalos de rosas, tallos de girasol y vástagos de plátano, un procedimiento general aplicado a cualquiera de los residuos orgánicos, tal y como se definió en el diagrama de flujo mostrado - figura 8-. Es importante aclarar que en caso de que el residuo no sea fibroso o poco rígido, como el caso de los pétalos de rosas, se debe hacer caso omiso al paso de la cocción alcalina, continuando con el proceso descrito.

El procesamiento de $5 \mathrm{~kg}$ de residuo orgánico generó $2 \mathrm{~kg}$ de pulpa que puede ser mezclada con diversas cantidades -entre $20 \%$ y $40 \%$ en masa- de pulpa de papel bond reciclado para obtener 44 hojas de papel después del proceso de secado, el cual se realiza sobre un sistema vertical de mallas de alambre que permite el paso del aire sin exposición directa de la luz solar para evitar la fotodegradación del color que, en el caso del papel artesanal vegetal, es un atractivo comercial. 


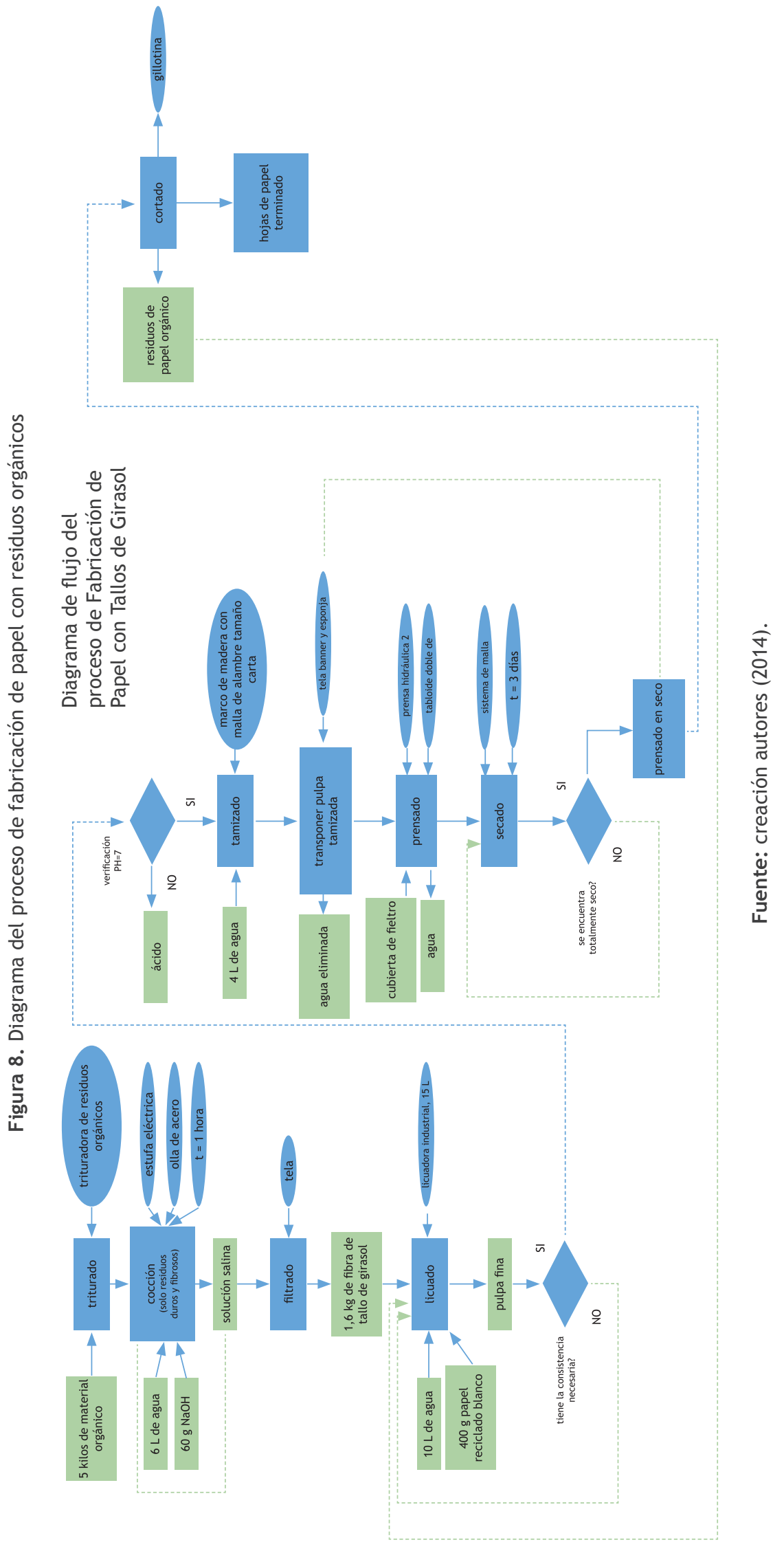


Pruebas de calidad: se fabricaron 200 hojas con diferentes porcentajes de pulpa vegetal y pulpa de papel blanco reciclado a fin de lograr establecer cuáles de ellos ofrecían mejores características de calidad.

El resultado de la comparación de los parámetros de calidad evaluados frente al papel bond se presentan en la tabla 1.

Tabla 1. Calificaciones para la valoración de los parámetros de calidad a tres porcentajes de adición de pulpa de papel reciclado

\begin{tabular}{|c|c|c|c|c|c|c|c|c|c|}
\hline \multirow[t]{2}{*}{ Evaluacion de papel } & \multicolumn{3}{|c|}{$\begin{array}{l}\text { Vastago de } \\
\text { platano }\end{array}$} & \multicolumn{3}{|c|}{\begin{tabular}{|c|}
$\begin{array}{c}\text { Tallos de } \\
\text { girasol }\end{array}$ \\
\end{tabular}} & \multicolumn{3}{|c|}{$\begin{array}{c}\text { Petalos de } \\
\text { rosa }\end{array}$} \\
\hline & $0 \%$ & $20 \%$ & $40 \%$ & $\mathbf{0} \%$ & $20 \%$ & $40 \%$ & $0 \%$ & $20 \%$ & $40 \%$ \\
\hline 1 Color & 5 & 3 & 2 & 2 & 3 & 2 & 5 & 3 & 2 \\
\hline 2 Estabilidad dimensional & 1 & 1 & 1 & 1 & 2 & 3 & 5 & 1 & 1 \\
\hline 3 Pliegue & 1 & 1 & 1 & 4 & 1 & 2 & 5 & 1 & 1 \\
\hline 4 Escritura & 3 & 2 & 2 & 3 & 2 & 3 & 5 & 1 & 1 \\
\hline 5 Humectacion al ambiente & 1 & 1 & 1 & 1 & 1 & 1 & 1 & 1 & 1 \\
\hline 6 Textura & 4 & 2 & 3 & 2 & 2 & 3 & 5 & 2 & 2 \\
\hline 7 Resistencia a la penetracion & 1 & 1 & 1 & 1 & 1 & 1 & 5 & 1 & 1 \\
\hline
\end{tabular}

Fuente: creación autores (2015).

Color: las tonalidades que se presentan en las hojas fabricadas con los diferentes residuos, vástago de plátano, tallo de girasol, pétalos de rosa, en porcentajes de $40 \%$ papel con $60 \%$ pulpa y $20 \%$ papel con $80 \%$ pulpa, presentan una coloración clara tomando como base el color del residuo empleado. Las hojas elaboradas con el $100 \%$ con pulpa del residuos vegetal, esto es, que tienen $0 \%$ de pulpa de papel reciclado, presentan un tono oscuro similar al del residuo orgánico original, exceptuando las hojas elaboradas con tallos de girasol las cuales presentan una tonalidad clara. Figura 9.

Figura 9. Gráfica de tonalidades de color obtenidas en el papel fabricado

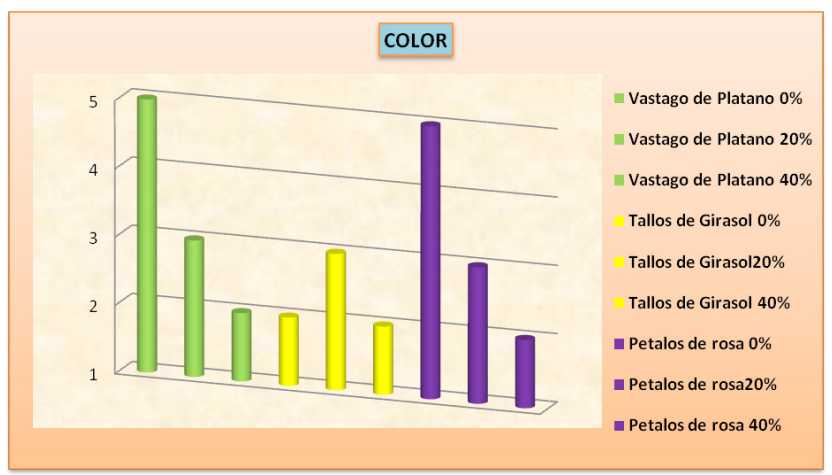

Fuente: creación autores (2015).
Estabilidad dimensional: evaluando este aspecto como la facilidad para dejarse doblar, en el que 1 es fácil de doblar y 5 no dobla, se obtuvo que de los tres residuos orgánicos evaluados en los tres porcentajes, $100 \%$ pulpa de residuo orgánico, 20\% papel reciclado y $80 \%$ pulpa, $40 \%$ papel reciclado y $60 \%$ pulpa, el que mejor respondió fue el vástago de plátano el cual en sus diferentes porcentajes presentó buena estabilidad dimensional ya que fue muy fácil de doblar. Por el contrario, los tallos de girasol con porcentajes de $20 \%$ papel, $80 \%$ pulpa, y $40 \%$ papel y $60 \%$ pulpa, presentan una pequeña resistencia al doblarlos; por último, las hojas elaboradas $100 \%$ con residuos de pétalos de rosa obtuvieron una calificación de 5 , no dobla, ya que no fue posible desprenderlas de la base donde se las ubicó inicialmente antes de pasar al proceso de secado. Esto se atribuye a la fragilidad y poca firmeza de los pétalos de rosas al conformar el papel. Figura 10.

Figura 10. Gráfica de evaluación de la estabilidad dimensional para las hojas fabricadas

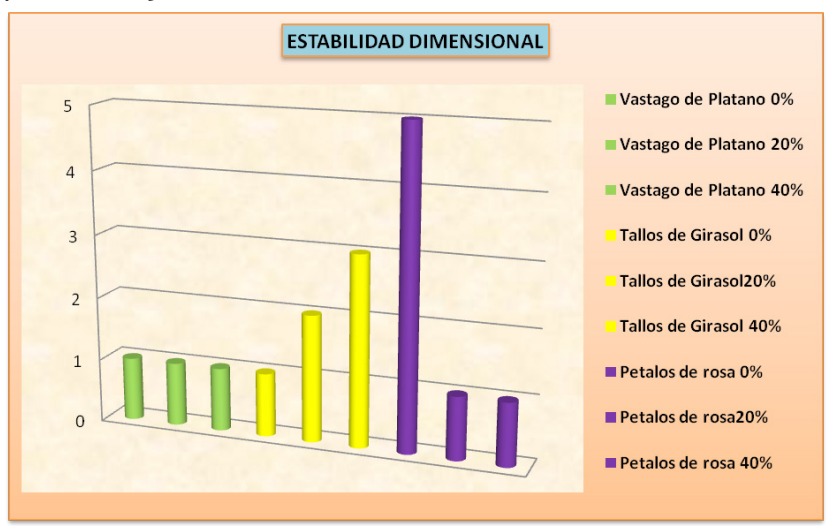

Fuente: creación autores (2015).

Pliegue: las hojas elaboradas con $100 \%$ tallos de girasol permiten ser dobladas; sin embargo, presentan una tendencia a romperse. Las hojas elaboradas $100 \%$ con residuos de pétalos de rosa obtuvieron una calificación de 5 , es decir, se rompen, ya que aunque no fue posible desprenderlas de la base donde se las ubicó inicialmente antes pasar al proceso de secado, se puede observar que la adherencia entre los pétalos no es consistente, por lo que se rompen de manera fácil debido a la poca cohesión presentada entre las fibras. Figura 11. 
Figura 11. Gráfica de la evaluación del pliegue para las hojas fabricadas

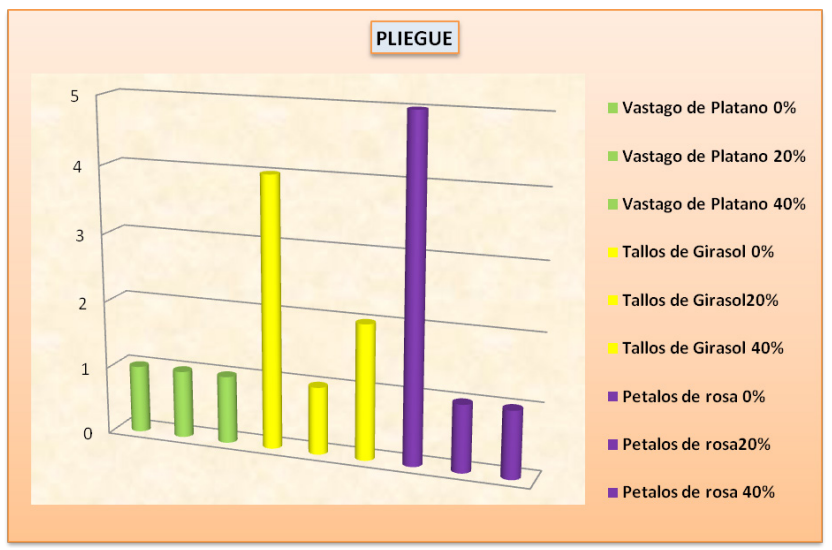

Fuente: creación autores (2015).

Humectación al ambiente: después de realizar el proceso de secado se observa que ninguna de las hojas elaboradas ganó peso significativo por adquirir humedad del ambiente o este fue mínimo, es decir, no presentan humedad significativa, lo cual define un rango entre $0-20 \%$. Figura 12.

Figura 12. Gráfica de la evaluación de la humectación al ambiente para las hojas fabricadas

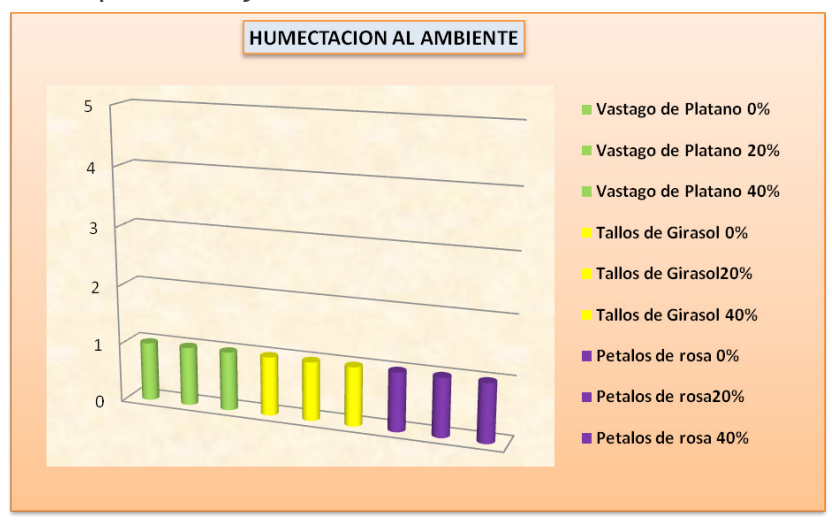

Fuente: creación autores (2015).

Textura: esta característica se evaluó palpando con la yema de los dedos su textura para establecer qué tan áspera es la hoja respecto a la textura presentada por la hoja de papel bond-usado como patrón de comparación-.

Las hojas elaboradas con un 100\% de pulpa de pétalos de rosa presentan una textura áspera, no obstante, las hojas que se obtuvieron con el $100 \%$ de pulpa de vástago de plátano presentan una textura rugosa inherente a la cohesión entre las fibras del mismo. Figura 13.
Figura 13. Gráfica de la evaluación de la textura para las hojas fabricadas

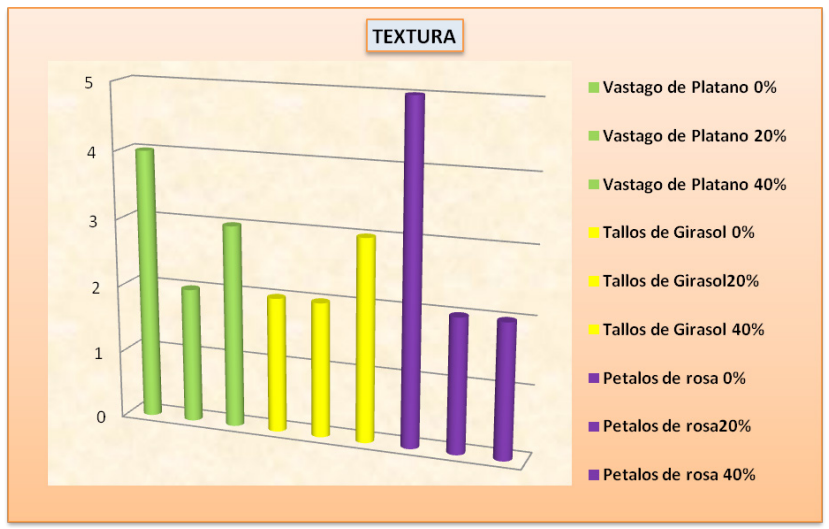

Fuente: creación autores (2015).

Escritura: las hojas fabricadas con pétalos de rosa en los diferentes porcentajes, excepto las que fueron elaboradas con el $100 \%$ de pétalos de rosa, permiten realizar una escritura suave que es similar a la realizada en una hoja en blanco de papel bond fabricada a partir de la fibra que posee la celulosa de los árboles.

Las hojas elaboradas con $100 \%$ de vástago de plátano permiten la escritura; sin embargo, no es tan legible como la que se presenta en las otras hojas fabricadas. De otro lado, las hojas elaboradas 100\% con pétalos de rosa no admiten ningún tipo de escritura puesto que la adherencia entre los pétalos no es óptima y el color de éstas es oscuro. Figura 14.

Figura 14. Gráfica de la evaluación de la escritura para las hojas fabricadas

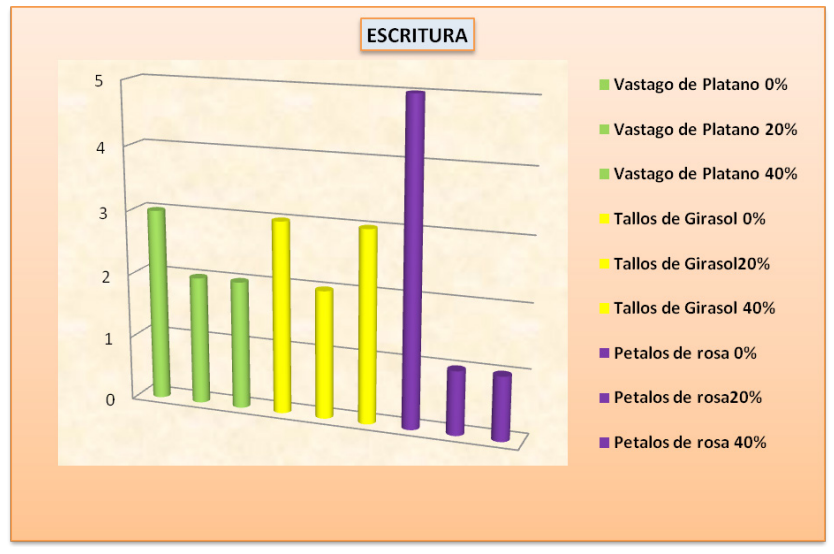

Fuente: creación autores (2015).

Resistencia a la penetración: las hojas fabricadas a partir de residuos como vástago de plátano, en un porcentaje de $100 \%$ pulpa de residuo, $20 \%$ 
pulpa de papel reciclado, $80 \%$ pulpa y $40 \%$ papel, $60 \%$ pulpa; tallos de girasol en un porcentaje de $100 \%$ pulpa, $20 \%$ papel $80 \%$ pulpa y $40 \%$ papel, $60 \%$ pulpa; y pétalos de rosa en un porcentaje de $20 \%$ papel $80 \%$ pulpa y $40 \%$ papel $60 \%$ pulpa, no son fáciles de penetrar-soportan peso de por lo menos $1 \mathrm{~kg}$ aproximadamente-; no obstante, las hojas elaboradas con el $100 \%$ de pétalos de rosa no soportan el peso y su penetración ocurre fácilmente por la falta de cohesión entre las fibras. Figura 15.

Figura 15. Gráfica de la evaluación de la resistencia a la penetración

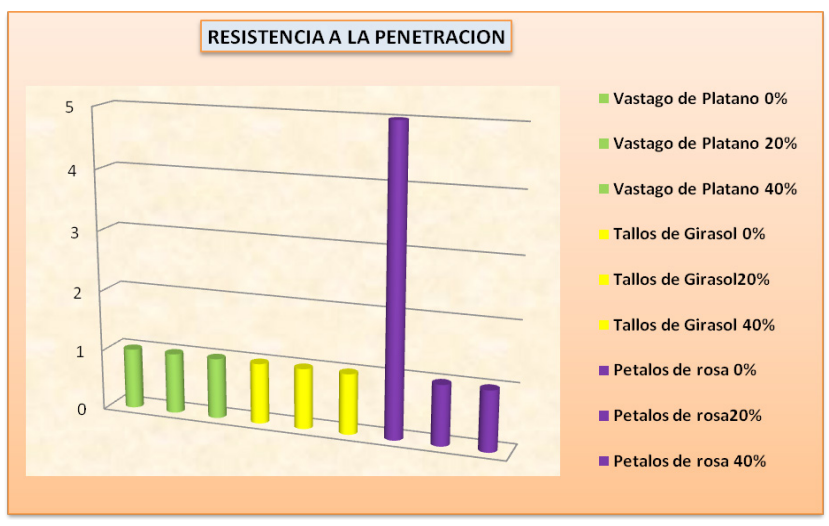

Fuente: creación autores (2015).

\section{CONCLUSIONES}

El proceso establecido para la fabricación de papel, que empleó residuos orgánicos de pétalos de rosas, tallos de girasol y vástago de plátano, permitió obtener cerca de 22 hojas de papel por cada kg de pulpa de residuo procesado y combinado con pulpa de papel blanco reciclado en porcentajes en masa del $20 \%$ y $40 \%$.

La calidad mostrada por las hojas de papel fabricadas con los residuos orgánicos de pétalos de rosas, tallos de girasol y vástago de plátano, fue evaluada en relación a los parámetros considerados en la metodología, encontrando una textura y color aceptables para el trabajo manual de empaques y tarjetería, resistentes a los cortes por pliegues, penetración y ganancia de humedad del ambiente. El papel que mejor se comportó frente a las pruebas de calidad fue el fabricado con la mezcla de la pulpa de cada residuo orgánico procesado mezclado con pulpa de papel reciclado, en porcentajes de $20 \%$ y $40 \%$ en masa.

El papel hecho con base en residuos orgánicos representa una ventaja competitiva para la industria debido a que emplea materiales reciclados-pétalos de rosa, tallo de girasol y vástago de plátanofáciles de conseguir y totalmente gratuitos, lo que hace que sus costos de producción sean bajos.

El consumo energético del proceso empleado para la fabricación del papel basado en residuos orgánicos es estimado en $0.6 \mathrm{KWh}$ considerando el uso de trituradora, licuadora y estufa para cocción alcalina.

El papel hecho con base en residuos orgánicos posee características que resaltan su color y textura por emplear fibras naturales que aportan resistencia y facilitan el trabajo manual de empaques y tarjetería.

El proceso descrito y el producto obtenido consideran un mínimo consumo de agua al reusar el agua de deshecho generada en el proceso de fabricación, un intento que sirve para fortalecer la gestión ambiental del consumo responsable, el aprovechamiento y la valorización de los residuos con potencial de recuperación; asimismo, aporta una alternativa innovadora dentro del crecimiento y competitividad empresarial al constituirse en una oportunidad de generación de empleo a bajo costo debido a los gastos de materia prima y operatividad del proceso.

El producto ofrecido no podría ser totalmente blanco pues esto requeriría de productos químicos que no son amigables con el ambiente y se generaría un mayor costo de producción.

\section{REFERENCIAS}

ANDI. (2013). Cámara de pulpa, papel y cartón. Colombia: ANDI.

Burgos, L. (2012). Elaboración de papel artesanal, su coloración y permanencia. España: AEA Editores.

Canché-Escamilla, G., De los Santos-Hernández, J., Andrade-Canto, S., \& Gómez-Cruz, R. (2005). Production of Cellulose from Banana Plant Agricultural Waste. Información Tecnológica, 16(1), 83-88.

DANE. (2012). Informe pobreza y desigualdad. Colombia: DANE.

Ervasti, I., Miranda, R., \& Kauranen, I. (2016). Paper recycling framework, the "Wheel of Fiber". Journal of Environmental Management(174), 35-44. 
González, A. (2005). Factibilidad de uso de fibra de raquis de palma aceitera para elaborar papel. Costa Rica: Universidad Earth.

Ministerio de Agricultura. (2008). Anuario estadístico del sector agropecuario.

Observatorio Colombiano de Ciencia y Tecnología. (2013). Indicadores de ciencia y tecnología de Colombia. Colombia: Observatorio Colombiano de Ciencia y Tecnología.

Otero, A. (2001). Medio ambiente y educación. Novedades educativas.

Pérez, R., Rojas, P., y Ordóñez, C. (2010). Desarrollo sostenible: principios, aplicaciones y lineamientos de política para Colombia. Santiago de Cali: Universidad del Valle.
Pintado, T., Joaquín, S., y Grande, I. (2010). Introducción a la investigación de mercados. España: Esıc.

Rodríguez, G., Londoño, B., y Herrera, G. (2008). Perspectivas del derecho ambiental en Colombia. Colombia: Universidad del Rosario.

Uaesp. (2013). Reporte anual. Bogotá.

Wang, L., Templer, R., \& Murphy, R. (2012). A Life Cycle Assessment (LCA) comparison of three management options for waste papers: Bioethanol production, recycling and incineration with energy recovery. Bioresource Technology(120), 89-98. 